\title{
ICTV Virus Taxonomy Profile: Thaspiviridae 2021
}

\author{
Jong-Geol Kim', Khaled S. Gazi², Mart Krupovic ${ }^{3}$, Sung-Keun Rhee ${ }^{2, *}$ and ICTV Report Consortium
}

\begin{abstract}
Members of the family Thaspiviridae have linear dsDNA genomes of 27 to $29 \mathrm{kbp}$ and are the first viruses known to infect mesophilic ammonia-oxidizing archaea of the phylum Thaumarchaeota. The spindle-shaped virions of Nitrosopumilus spindle-shaped virus 1 possess short tails at one pole and measure $64 \pm 3 \mathrm{~nm}$ in diameter and $112 \pm 6 \mathrm{~nm}$ in length. This morphology is similar to that of members of the families Fuselloviridae and Halspiviridae. Virus replication is not lytic but leads to growth inhibition of the host. This is a summary of the International Committee on Taxonomy of Viruses (ICTV) Report on the family Thaspiviridae, which is available at ictv.global/report/thaspiviridae.
\end{abstract}

Table 1. Characteristics of members of the family Thaspiviridae

\begin{tabular}{|ll|}
\hline Example: & Nitrosopumilus spindle-shaped virus 1 (MK570053), species Nitmarvirus NSV1, genus Nitmarvirus \\
\hline Virion & Spindle-shaped, measuring $64 \pm 3 \mathrm{~nm}$ in diameter and $112 \pm 6 \mathrm{~nm}$ in length, with short fibres at one pole. \\
Genome & Linear dsDNA $(27-29 \mathrm{kbp})$ with $176 \mathrm{bp}$ terminal inverted repeats \\
Replication & Non-lytic, chronic infection. Protein-primed family B DNA polymerase is involved in viral DNA replication \\
Translation & Not characterized \\
Host range & Ammonia-oxidizing archaea of the genus Nitrosopumilus \\
Taxonomy & Single genus with one species \\
\hline
\end{tabular}

\section{VIRION}

The virion of Nitrosopumilus spindle-shaped virus 1 is $64 \pm 3 \mathrm{~nm}$ in diameter and $112 \pm 6 \mathrm{~nm}$ in length with a short tail at one pole (Table 1, Fig. 1). The predicted major capsid protein (81 amino acids) contains two highly hydrophobic $\alpha$-helical regions. The spindle-shape morphology of the virion is very similar to those of members of the families Fuselloviridae [1] and Halspiviridae [2], which infect hyperthermophilic and hyperhalophilic archaea, respectively. When associated with the host cells, some virions are observed as elongated structures, with long thin tails connected to the cell surface $[3,4]$.

\section{GENOME}

The genome of Nitrosopumilus spindle-shaped virus 1 is linear dsDNA (27-29 kbp), terminating with $176 \mathrm{bp}$ inverted repeats (Fig. 2). The virus genome is predicted to carry 48 genes. With one exception, the proteins predicted to be encoded by Nitrosopumilus spindle-shaped virus 1 are unrelated to those of other archaeal and bacterial viruses. The exception, protein-primed B DNA polymerase, is also found in several groups of archaeal viruses and non-viral mobile genetic elements which, similar to Nitrosopumilus spindle-shaped virus 1, have linear genomes with terminal inverted repeats [4].

\section{REPLICATION}

The virus establishes a chronic infection and its replication is not lytic, with virions being continuously extruded into the environment [4]. The virus genome is likely to be replicated by the virus-encoded protein-primed family B DNA polymerase, as has been inferred for haloarchaeal halspiviruses [2] and crenarchaeal ampullaviruses [5]. In addition, the virus encodes

Received 15 June 2021; Accepted 15 June 2021; Published 30 July 2021

Author affiliations: 'Department of Biological Sciences, Wonkwang University, Iksan, 54538, Republic of Korea; ${ }^{2}$ Department of Biological Sciences and Biotechnology, Chungbuk National University, Cheongju 28644, Republic of Korea; ${ }^{3}$ Archaeal Virology Unit, Institut Pasteur, Paris 75015, France.

${ }^{*}$ Correspondence: Sung-Keun Rhee, rhees@cbnu.ac.kr

Keywords: ICTV Report; taxonomy; Thaspiviridae.

001631 (c) 2021 

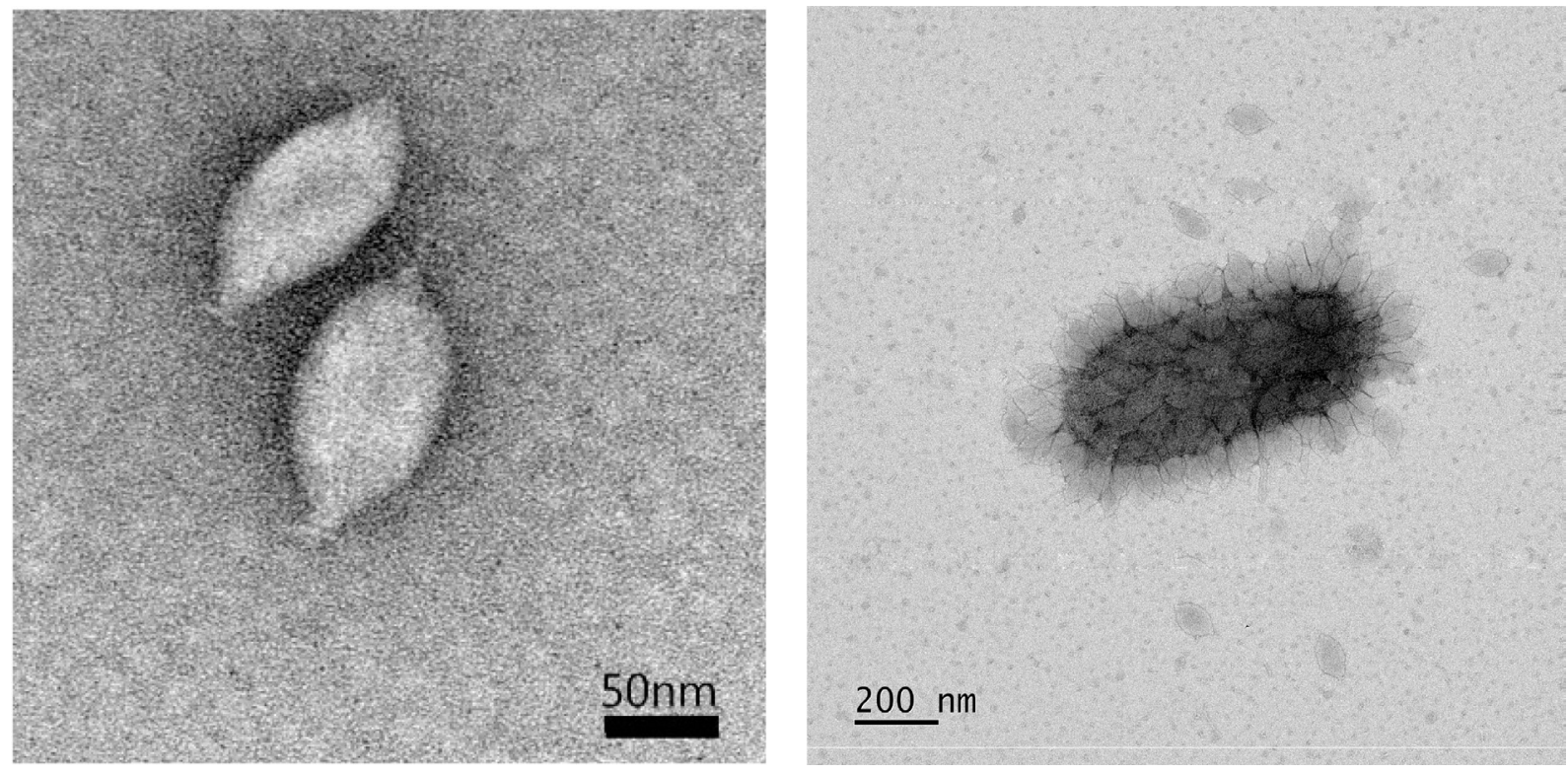

Fig. 1. (Left) Transmission electron micrograph of negatively-stained virions of Nitrosopumilus spindle-shaped virus 1. (Right) Virus particles attached to the surface of a host cell.

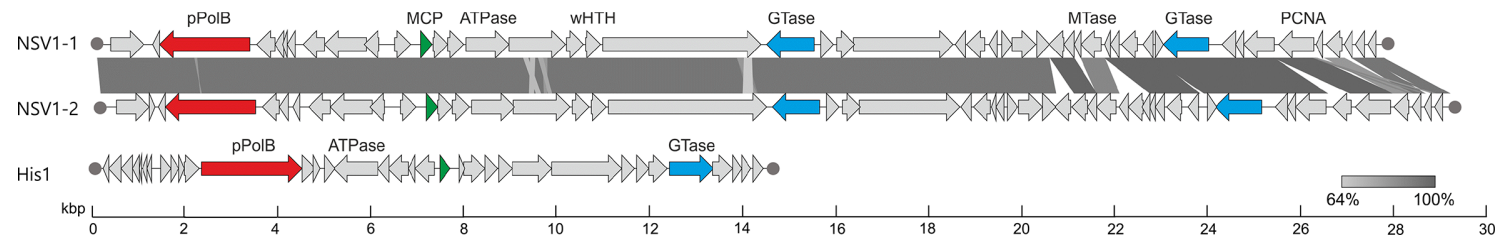

Fig. 2. Genome maps of Nitrosopumilus spindle-shaped virus 1 isolates NSV1-1 and NSV1-2, and of the haloarchaeal halspivirus His1. Shared ORFs are connected by shaded areas based on sequence identity. Functionally equivalent (but not necessarily homologous) genes are indicated with matching colours. Filled circles indicate terminal inverted repeats. pPolB, protein-primed family B DNA polymerase; MCP, major capsid protein (putative); wHTH, winged helix-turn-helix; GTase, glycosyltransferase; MTase, DNA methyltransferase; PCNA, proliferating cell nuclear antigen.

a proliferating cell nuclear antigen which is also likely to be involved in viral genome replication.

\section{TAXONOMY}

Current taxonomy: ictv.global/taxonomy. The single genus Nitmarvirus includes one species Nitmarvirus NSV1.

\section{RESOURCES}

Full ICTV Report on the family Thaspiviridae: ictv.global/ report/thaspiviridae.

\section{Funding information}

Production of this Profile, the ICTV Report, and associated resources was funded by a grant from the Wellcome Trust (WT108418AIA) and the National Research Foundation of Korea (NRF) grants funded by the Ministry of Science, ICT \& Future Planning (2021R1A2C3004015).

\section{Acknowledgements}

Members of the ICTV Report Consortium are Stuart G. Siddell, Elliot J. Lefkowitz, Sead Sabanadzovic, Peter Simmonds, F. Murilo Zerbini, Donald B. Smith, Richard J. Orton and Mart Krupovic.

Conflicts of interest

The authors declare that there are no conflicts of interest.

\section{References}

1. Krupovic M, Quemin ER, Bamford DH, Forterre P. Prangishvili D. Unification of the globally distributed spindle-shaped viruses of the Archaea. J Virol 2014;88:2354-2358.

2. Bath C, Cukalac T, Porter K, Dyall-Smith ML. His1 and His2 are distantly related, spindle-shaped haloviruses belonging to the novel virus group, Salterprovirus. Virology 2006;350:228-239.

3. Häring M, Vestergaard G, Rachel R, Chen L, Garrett RA, et al. Virology: independent virus development outside a host. Nature 2005;436:1101-1102.

4. Kim JG, Kim SJ, Cvirkaite-Krupovic V, WJ Y, Gwak JH, et al. Spindleshaped viruses infect marine ammonia-oxidizing thaumarchaea. Proc Natl Acad Sci U S A 2019;116:15645-15650.

5. Prangishvili D, Krupovic M, ICTV Report Consortium. ICTV Virus Taxonomy Profile: Ampullaviridae. J Gen Virol 2018:99:288-289. 